\title{
Predicting ambient aerosol thermal-optical reflectance measurements from infrared spectra: elemental carbon
}

\author{
A. M. Dillner ${ }^{1}$ and S. Takahama ${ }^{2}$ \\ ${ }^{1}$ University of California - Davis, Davis, California, USA \\ ${ }^{2}$ École polytechnique fédérale de Lausanne, Lausanne, Switzerland
}

Correspondence to: A. M. Dillner (amdillner@ucdavis.edu) and S. Takahama (satoshi.takahama@epfl.ch)

Received: 18 March 2015 - Published in Atmos. Meas. Tech. Discuss.: 23 June 2015

Revised: 14 September 2015 - Accepted: 17 September 2015 - Published: 2 October 2015

\begin{abstract}
Elemental carbon (EC) is an important constituent of atmospheric particulate matter because it absorbs solar radiation influencing climate and visibility and it adversely affects human health. The EC measured by thermal methods such as thermal-optical reflectance (TOR) is operationally defined as the carbon that volatilizes from quartz filter samples at elevated temperatures in the presence of oxygen. Here, methods are presented to accurately predict TOR EC using Fourier transform infrared (FT-IR) absorbance spectra from atmospheric particulate matter collected on polytetrafluoroethylene (PTFE or Teflon) filters. This method is similar to the procedure developed for OC in prior work (Dillner and Takahama, 2015). Transmittance FT-IR analysis is rapid, inexpensive and nondestructive to the PTFE filter samples which are routinely collected for mass and elemental analysis in monitoring networks. FT-IR absorbance spectra are obtained from 794 filter samples from seven Interagency Monitoring of PROtected Visual Environment (IMPROVE) sites collected during 2011. Partial least squares regression is used to calibrate sample FT-IR absorbance spectra to collocated TOR EC measurements. The FT-IR spectra are divided into calibration and test sets. Two calibrations are developed: one developed from uniform distribution of samples across the EC mass range (Uniform EC) and one developed from a uniform distribution of Low EC mass samples (EC $<2.4 \mu \mathrm{g}$, Low Uniform EC). A hybrid approach which applies the Low EC calibration to Low EC samples and the Uniform EC calibration to all other samples is used to produce predictions for Low EC samples that have mean error on par with parallel TOR EC samples in the same mass range and an estimate of the minimum detection limit (MDL) that is on par with TOR EC MDL. For all samples, this hybrid
\end{abstract}

approach leads to precise and accurate TOR EC predictions by FT-IR as indicated by high coefficient of determination $\left(R^{2} ; 0.96\right)$, no bias $\left(0.00 \mu \mathrm{g} \mathrm{m}^{-3}\right.$, a concentration value based on the nominal IMPROVE sample volume of $32.8 \mathrm{~m}^{3}$ ), low error $\left(0.03 \mu \mathrm{g} \mathrm{m}^{-3}\right)$ and reasonable normalized error $(21 \%)$. These performance metrics can be achieved with various degrees of spectral pretreatment (e.g., including or excluding substrate contributions to the absorbances) and are comparable in precision and accuracy to collocated TOR measurements. Only the normalized error is higher for the FT-IR EC measurements than for collocated TOR. FT-IR spectra are also divided into calibration and test sets by the ratios OC/EC and ammonium/EC to determine the impact of OC and ammonium on EC prediction. We conclude that FT-IR analysis with partial least squares regression is a robust method for accurately predicting TOR EC in IMPROVE network samples, providing complementary information to TOR OC predictions (Dillner and Takahama, 2015) and the organic functional group composition and organic matter estimated previously from the same set of sample spectra (Ruthenburg et al., 2014).

\section{Introduction}

Elemental carbon (EC) in atmospheric aerosols adversely impacts human health (Janssen et al., 2011) and contributes to climate warming (Bond et al., 2013) and decreased visibility (Hand et al., 2014). Elemental carbon is measured by large monitoring networks such as the Interagency Monitoring of PROtected Visual Environments (IMPROVE) network (Hand et al., 2012; Malm et al., 1994) in rural ar- 
eas of the USA, the Speciation Trends Network/Chemical Speciation Network (Flanagan et al., 2006) in urban areas of the USA, and the European Monitoring and Evaluation Programme (EMEP; Torseth et al., 2012) throughout Europe. These regional multi-year data sets are useful for observing trends in particulate concentrations (Hand et al., 2013; Hidy et al., 2014; Torseth et al., 2012) and visibility (Hand et al., 2014), evaluating aerosol transport models (Mao et al., 2011), constraining climate models (Liu et al., 2012) and assessing health impacts (Krall et al., 2013). EC is measured using thermal-optical methods such as thermaloptical reflectance (TOR) (Chow et al., 2007), NIOSH 5040 (Birch and Cary, 1996) and European Supersites for Atmospheric Aerosol Research (EUSAAR-2 protocol; Cavalli et al., 2010), in which particles collected on quartz filters are subjected to a temperature gradient, first in an inert environment and then in an oxidizing environment (Chow et al., 2007). Organic carbon (OC) and EC are operationally defined by the temperature and environmental conditions under which the carbon evolves from the aerosol sample. Charred organic material is subtracted from the measured EC based on laser reflectance or transmittance (Cavalli et al., 2010; Chow et al., 2007). These thermal-optical methods are not applicable to the PTFE (polytetrafluoroethylene) media used for gravimetric mass, elemental composition and sometimes light absorption in sampling networks because the filter material is unstable at high temperatures. TOR methods are also destructive to the sample and expensive.

Thermal-optical methods are one type of method that seeks to measure carbon in atmospheric aerosols that is structurally similar to graphite in that it is composed of $s p^{2}$ bonds and is strongly light absorbing (Bond and Bergstrom, 2006). Thermal-optical methods refer to this material as EC. Other methods use light absorption to characterize $s p^{2}$-bonded carbon in aerosol (Andreae and Gelencsér, 2006) and typically refer to the constituent being measured as black carbon (BC). Continuous light absorption measurements are made by such instruments as the PSAP (Bond et al., 1999), aethalometer (Collaud Coen et al., 2010) and multi-angle absorption photometer (Petzold et al., 2005). Time-integrated absorption measurements are made from filter samples using instruments such as the hybrid-integrating plate method used by the IMPROVE network (White et al., 2015). Fourier transform infrared (FT-IR) spectroscopy has also been used to characterize $s p^{2}$-bonded carbon in particles and other environmental samples. FT-IR spectra of ground graphite and activated carbon have prominent peaks at $1580 \mathrm{~cm}^{-1}$ which were assigned to the graphitic structure of the material (Friedel and Carlson, 1971). FT-IR spectra of ground charcoal and synthetic and marine sediments had similar peaks at $1580 \mathrm{~cm}^{-1}$ and additional peaks at 1720 and $1240 \mathrm{~cm}^{-1}$, which were assigned to carbonyl and single carbon-oxygen bonds, respectively (Smith et al., 1975). FT-IR and partial least squares regression (PLSR) have been used to quantify $\mathrm{BC}$ in soil samples by measuring benzene polycarboxylic acids, which are aromatic markers for black carbon (Bornemann et al., 2007). In a companion paper (Takahama et al., 2015), we will discuss similarities and differences in the vibrational modes identified by these previous authors relevant for quantitative prediction in atmospheric elemental carbon. However, the presence of wave numbers that correlate to TOR EC indicates the potential for FT-IR spectra calibrated to TOR EC using PLSR to predict TOR EC in aerosol samples.

In this work, FT-IR spectra are calibrated to TOR EC using PLSR, similar to our previous method for predicting TOR OC (Dillner and Takahama, 2015). PLSR is a general method that has been used to calibrate FT-IR spectra of environmental samples such as dust (Weakley et al., 2014), food (Polshin et al., 2011) and soil (Bornemann et al., 2007) to constituents of interest. As described above, thermal-optical methods currently provide $\mathrm{EC}$ measurements in air monitoring network ambient particle matter samples. Such networks simultaneously sample particles on PTFE filters which are used for gravimetric mass and elemental composition analysis and can also be used to obtain FT-IR spectra. In this work, EC is predicted from infrared spectra of PTFE filter samples of aerosols using PLSR. The methods are developed and tested using EC from TOR analysis and FT-IR spectra from parallel PTFE filters from 1 year of samples from seven IMPROVE sites.

The objective of this work is to demonstrate the feasibility of predicting TOR EC from infrared spectra as a second step in developing an inexpensive, fast and nondestructive method for carbon measurements in particulate matter (PM) monitoring networks. Prediction of TOR OC was the first step in developing this method and was established in Dillner and Takahama (2015). Sampling networks which only collect samples on PTFE filters, such as the Federal Reference Method network used for compliance with National Ambient Air Quality Standards for PM mass concentrations in the United States, can also use the FT-IR and PLSR method presented here and in Dillner and Takahama (2015) to obtain information about the carbonaceous aerosol provided that the samples have aerosol composition similar to the calibration samples. In this work, we will show that the prediction of TOR EC can be accomplished with accuracy on par with TOR EC measurement precision. Furthermore, we will mechanistically explain important differences in sample composition between calibration and test sets that can lead to increased prediction errors; for this we use additional IMPROVE measurements to aid in our interpretation. Finally, we will demonstrate how sensitivity to sample composition is manifested in predictions for sites that are not included in the calibration set. 


\section{Methods}

\subsection{IMPROVE network samples}

This study uses 794 IMPROVE particulate matter samples collected on PTFE filters and 54 blank PTFE filters. The IMPROVE samples were collected at seven sites during 2011 (Fig. S1 in the Supplement). These are the same samples, blanks and consequent FT-IR spectra used for developing the OC method in Dillner and Takahama (2015) and the organic matter/organic carbon method in Ruthenburg et al. (2013). Additional details are provided in these papers. In the IMPROVE network, filter samples of particles less than $2.5 \mu \mathrm{m}$ $\left(\mathrm{PM}_{2.5}\right)$ are collected every third day from midnight to midnight local time at a nominal flow rate of 22.8 liters per minute, which yields a nominal volume of $32.8 \mathrm{~m}^{3}$.

The FT-IR analysis is applied to $25 \mathrm{~mm}$ PTFE filters (Teflon, Pall Gelman, $3.53 \mathrm{~cm}^{2}$ sample area) that are analyzed for gravimetric mass, elements and light absorption in the IMPROVE network. Quartz filters collected in parallel with the PTFE filters are analyzed by TOR and adjusted to account for charring of organic material prior to reporting EC mass in the IMPROVE network (Chow et al., 2007). For this work, the EC values are also adjusted to account for flow differences between the quartz and PTFE filters.

In order to provide reference performance metrics for the evaluation of the FT-IR to TOR comparisons (see Sect. 2.4 for a description of the metrics), measurements from seven IMPROVE sites with collocated TOR measurements (Everglades, Florida; Hercules Glade, Missouri; Hoover, California; Medicine Lake, Montana; Phoenix, Arizona; Saguaro West, Arizona; Seney, Michigan) are used.

\subsection{FT-IR analysis}

\subsubsection{Spectra acquisition}

PTFE filters are analyzed using a Tensor 27 FT-IR spectrometer (Bruker Optics, Billerica, MA) equipped with a liquid nitrogen-cooled wide-band mercury cadmium telluride detector. The samples are analyzed using transmission FTIR over the mid-infrared wave number region of 4000 to $420 \mathrm{~cm}^{-1}$ (Ruthenburg et al. (2014) describes the protocol in further detail). Absorbance spectra are calculated using a recent spectrum of the empty sample compartment as a zero reference. Air free of water vapor and carbon dioxide (delivered by purge-gas generator; PureGas LLC, Broomfield, $\mathrm{CO}$ ) is used to continuously purge the optical compartments of the instrument and to purge the sample compartment for $4 \mathrm{~min}$ before each sample or reference spectrum is acquired.

\subsubsection{Spectra preparation}

Three different versions of the absorption spectra are used in our analysis (Fig. S2), corresponding to different pretreatments and wavelength selection. (1) "Raw" spectra are unmodified spectra except that values interpolated during the zero-filling process are removed. These spectra contain all 2784 wave numbers. (2) "Baseline-corrected" spectra include absorbances above $1500 \mathrm{~cm}^{-1}$ and the substrate contribution is removed by subtracting an average blank filter spectrum and then using linear or polynomial baselines by spectral region as described by Takahama et al. (2013). These spectra are standardized to a $2 \mathrm{~cm}^{-1}$ resolution and so contain 1563 wave numbers. (3) "Truncated" spectra are the raw spectra interpolated to match the wave numbers in the baseline-corrected spectra, which excludes the PTFE peaks (the region below $1500 \mathrm{~cm}^{-1}$ ) and so also contain 1563 wave numbers.

\subsection{Calibration}

The FT-IR spectra are calibrated to TOR EC measurements using PLSR using the kernel pls algorithm, implemented by the pls library (Mevik and Wehrens, 2007) for the R statistical package (R Core Team, 2014). Conceptual description of PLSR can be found in Dillner and Takahama (2015), Takahama et al. (2015) and Ruthenburg et al. (2014) and references therein. Briefly, in PLSR the matrix of spectra is decomposed into factors and their respective weights. Candidate models are generated by varying the number of factors used to reconstruct the EC mass in the calibration filters.

Using the common approach for model selection and assessment (Hastie et al., 2009; Bishop, 2006; Witten et al., 2011), two-thirds of the 794 samples and two-thirds of the blanks filters (which are assumed to have 0 EC mass) are used for developing the calibration and one-third of the samples and blanks (called the test set) are used to evaluate the model. Ambient samples with EC below TOR EC MDL are excluded from the model so as not to train the calibration with samples that have a low signal-to-noise ratio. $K$-fold cross validation with $k=10$ is used to estimate the accuracy of each candidate model. The minimum root mean square error of cross validation (Mevik and Cederkvist, 2004) is used to select the model with the least prediction error. This procedure permits development and selection of PLSR models using only the samples in the calibration set and guards against over-fitting to a single set of samples. The test set, which has not been used in model development or selection, is used for model evaluation.

The above procedures are the same as those used to develop the OC calibration (Dillner and Takahama, 2015). For EC, possibly on account of lower mass concentrations, additional methods for including blanks into the calibration are needed. First, a calibration is developed without blank filters and used to predict the mass of EC on each blank filter. The blanks are then ordered by EC mass and every third is included in the test set and the remaining are put into the calibration set so that there are similar distributions (both positive and negative) of blanks in the two sets. Blanks are interspersed in the calibration at regular intervals so that each 


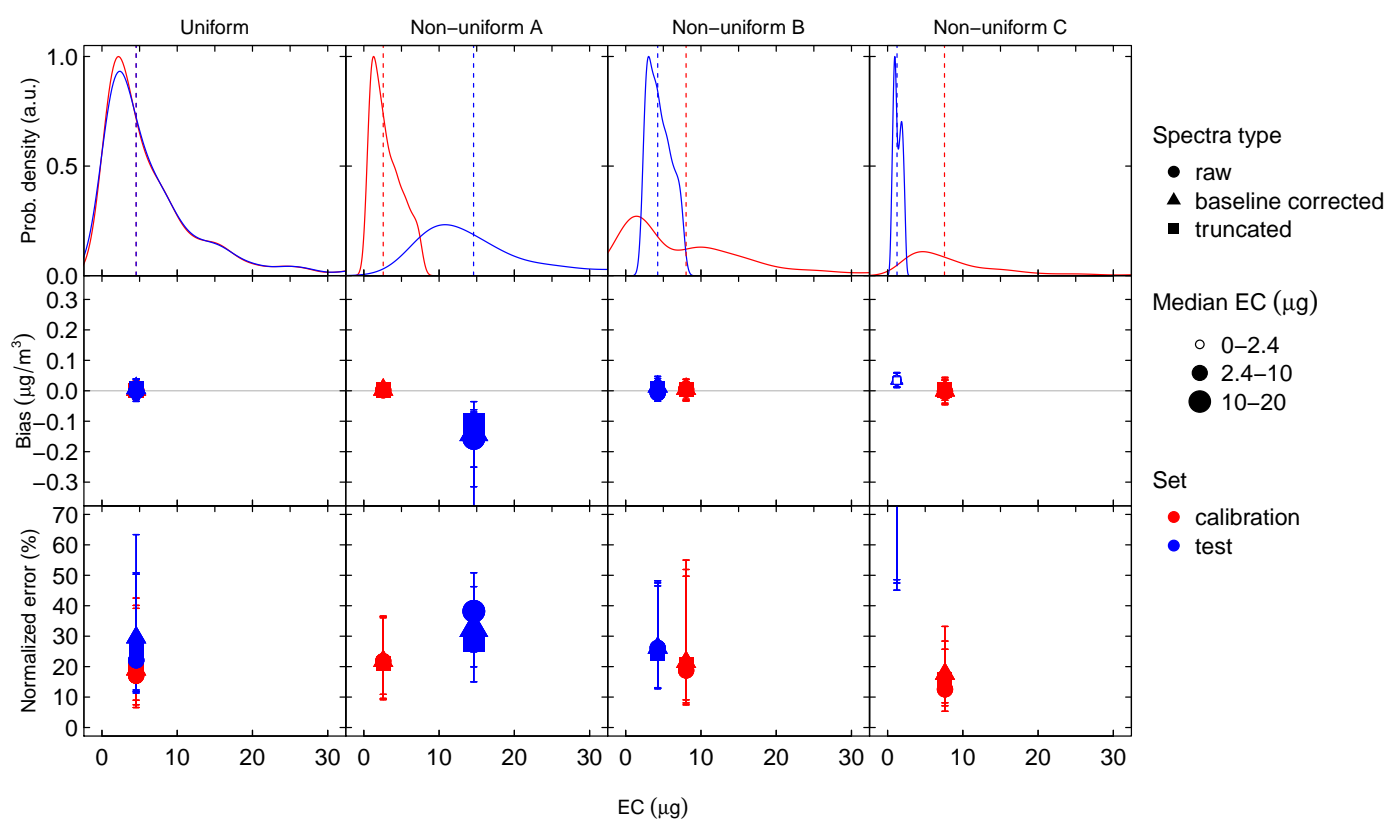

Figure 1. Uniform and Nonuniform EC calibrations. The probability density distribution of EC and bias and normalized error (with the interquartile range shown by error bars) in the calibration (red) and test (blue) sets for the Uniform EC case and three Nonuniform EC cases. Vertical lines are the median of the EC mass distributions color-coded for calibration and test sets.

iteration of the cross-validation process has a roughly consistent number of blanks used for calibration. Calibrations developed without ordering the blanks and without interspersing the blanks in the calibration filter set lead to inconsistent MDL values.

EC is predicted for a sample $(i)$ by taking the product of the absorbance at wave number $j\left(x_{i, j}\right)$ and the calibration vector $\left(\boldsymbol{b}_{j}\right)$ as shown in Eq. (1).

$\mathrm{EC}_{i}=\sum_{j} x_{i, j} \boldsymbol{b}_{j}$

Blank samples in the test set are used to calculate the MDL.

Multiple calibrations are developed by varying the spectra type used and by selecting filters for the calibration and test sets using different ordering regimes. Initially, we develop a set of calibrations based on uniform and nonuniform distributions of EC in the calibration and test sets. For the Uniform case, the samples are ordered by EC mass and every third sample is put into the test set so the distribution of EC is the same in the calibration and test sets. Three Nonuniform cases are developed to assess the impact of EC distribution on the quality of the calibration. For the Nonuniform cases, the samples are ordered by EC mass and then selected for calibration and test set based on where each samples falls within the range of EC masses. The three Nonuniform cases are: (1) samples with mass in the highest two-thirds of the EC mass range are used for calibration and samples with EC in the lowest third of the EC mass range are used as the test set (Nonuniform A); (2) samples in the highest and lowest third of the EC mass range are used to predict samples in the middle third of the EC mass range (Nonuniform B); and (3) samples with EC mass in the lowest two-thirds of the EC range are used to predict samples in the highest third of the EC mass range (Nonuniform C).

Figure 1 shows the Uniform and three Nonuniform calibrations developed for EC. A description of the performance metrics shown in Fig. 1 are given in Sect. 2.4. The top row gives the EC distribution of the test and calibration set for each case. The EC distributions reflect the algorithm used to select the filters for that case. The median and 25th to 75 th percentiles (interquartile range) of the bias and normalized error are shown in the lower two rows of Fig. 1 for each of the three spectra types (indicated by symbol shape). Small open symbols are used for sets with low median EC masses. Larger closed symbols have higher median EC masses. The EC distributions for the Uniform case shows that the distribution of samples in the calibration set and test set are very similar. This leads to predictions with small bias (middle plot) and median normalized errors (bottom plot) ranging from 15 to $30 \%$ for the test and calibration sets for the three spectral treatments. However, when low EC mass samples are used to predict high EC samples (Nonuniform A) and high samples are used to predict low EC samples (Nonuniform C), the test set is biased, leading to high error, especially when the EC values are low (Nonuniform C).

In order to eliminate bias in the calibration and improve prediction capability for Low EC samples, we develop a localized calibration for samples in the lowest third of the EC mass range $(\mathrm{EC}<2.4 \mu \mathrm{g})$. The Low Uniform EC calibration (or Low EC calibration) uses samples in the calibration set 
that are in the lowest third of the EC mass range to predict samples in the test set that are also in the lowest third of the EC mass range. Localization of the calibration is a commonly used method to improve the performance of the calibration, often at the more difficult to measure low end of the range. The Uniform (which contains samples across the whole range of EC; Fig. 1) and Low EC calibrations are used to predict Low EC test set samples and the resulting error and MDL are compared in Fig. 2. The mean error or precision of collocated TOR EC samples below $2.4 \mu \mathrm{g}$ and the reported TOR EC MDL are also shown in Fig. 2. The Low EC calibration reduces the error for all three spectral types to a value similar to that of the precision of collocated TOR EC samples in the same mass range. In addition, the MDL (which is based on the prediction of blank filters) is reduced to approximately the TOR MDL for all three spectral types. This comparison indicates that a localized calibration greatly improves the prediction quality for Low EC samples and that the error in the FT-IR prediction is due primarily to TOR EC measurement uncertainty.

A hybrid calibration method, which includes the Uniform calibration for the whole EC range and the Low EC calibration for the Low EC range is used for all results presented in Sect. 3, regardless of the ordering scheme used to select filters for the calibration and test sets. In the hybrid calibration method, the Uniform calibration is used to predict all filters in the test set. Those filters in the test set which are predicted to be in the lowest third of the EC mass are then analyzed by the Low EC calibration. The prediction from the full calibration is used for samples above the lowest third and predictions from the Low EC calibration are used for the Low EC samples.

A "Base case" hybrid calibration, in which the samples are chronologically stratified per site (i.e., ordered by date for each site), is developed as a reference scenario. Every third sample in the ordered list is put into the test set and the rest are put into the calibration set. The Base case has the same samples in the test set as the Base case for the OC predictions reported earlier (Dillner and Takahama, 2015). Description of a minor error in the $\mathrm{OC}$ calibration set for the Base case is documented in Sect. S3, and this has been corrected for the EC calculations in this work. The blanks are calculated and distributed as previously stated. This ordered set of samples based on sites and date provides fairly uniform distribution of $\mathrm{EC}$ and aerosol composition in the calibration and test sets. In addition to the Base case, Uniform and Nonuniform OC/EC, ammonium/EC and site-specific calibrations are developed using the hybrid approach and discussed in more detail in the Sect. 3.

\subsection{Methods for evaluating of the quality of calibration}

The quality of each calibration is evaluated by calculating four performance metrics: bias, error, normalized error and the coefficient of determination $\left(R^{2}\right)$ of the linear regression

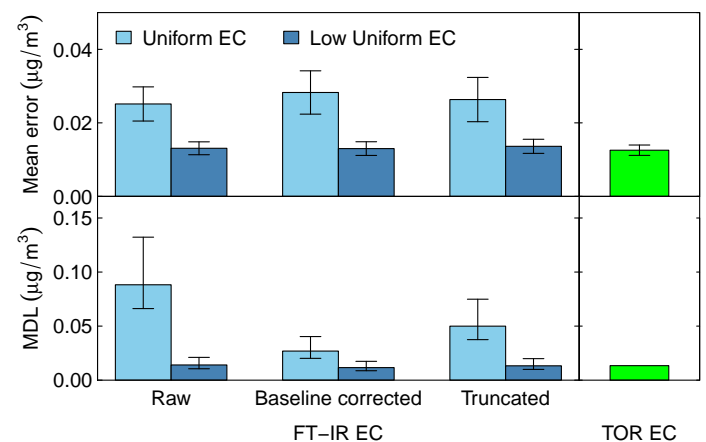

Figure 2. Mean error of a Low EC test set $(\mathrm{EC}<2.4 \mu \mathrm{g})$ and MDL from the Uniform EC and Low Uniform EC calibrations. The same Low EC test set is predicted by the Uniform and Low Uniform calibrations. The mean error of collocated EC samples less than $2.4 \mu \mathrm{g}$ and the reported EC MDL for the TOR method are shown for comparison.

fit of the predicted FT-IR EC to measured TOR EC. FT-IR EC is the EC predicted from the FT-IR spectra and the PLSR calibration model. The bias is the median difference between measured (TOR EC) and predicted FT-IR EC for the test set. Error is the median absolute bias. The normalized error for a single prediction is the error divided by the TOR EC value. The median normalized error is reported. The performance metrics are also calculated for the collocated TOR observations and compared to those of the FT-IR EC to TOR EC regression. The MDL and precision of the FT-IR method are calculated and compared to the reported MDL and calculated precision of the TOR method. The MDL of the FT-IR method is 3 times the standard deviation of the blanks in the test set (18 blank filters). MDL for the TOR method is 3 times the standard deviation of 514 blanks (Desert Research Intitute, 2012). Precision for both FT-IR and TOR are calculated using the 14 parallel samples in the test set at the Phoenix, AZ, site.

\section{Results}

\subsection{Predicting TOR EC from infrared spectra}

Using the hybrid calibration with the Base case scenario, Fig. 3 shows the prediction of the calibration samples and the test set samples along with the collocated TOR samples with the same EC distribution. The calibration and test sets are predicted with no bias (nonlinearity is removed by using the Low EC calibration) and have similar error, normalized error and $R^{2}$. The precision between TOR samples is expected to be better than the error between FT-IR EC and TOR EC because the TOR samples are collected on the same filter type and analyzed by the same method and as expected the normalized error is lower for the collocated TOR EC. However, the error between TOR EC and FT-IR EC is the same as the 
Table 1. MDL and precision for Hybrid FT-IR EC and TOR EC.

\begin{tabular}{lllll}
\hline & TOR-EC & $\begin{array}{l}\text { Hybrid } \\
\text { FT-IR EC } \\
\text { raw spectra }\end{array}$ & $\begin{array}{l}\text { Hybrid } \\
\text { FT-IR EC } \\
\text { baseline } \\
\text { corrected } \\
\text { spectra }\end{array}$ & $\begin{array}{l}\text { Hybrid } \\
\text { FT-IR EC } \\
\text { truncated } \\
\text { spectra }\end{array}$ \\
\hline MDL $\left(\mu \mathrm{g} \mathrm{m}^{-3}\right)^{\mathrm{a}}$ & $0.01^{\mathrm{b}}$ & 0.02 & 0.01 & 0.02 \\
$\%$ below MDL & 3 & 1 & 2 & 3 \\
Precision $\left(\mu \mathrm{g} \mathrm{m}^{-3}\right)^{\mathrm{a}}$ & 0.11 & 0.04 & 0.06 & 0.06 \\
Mean blank $(\mu \mathrm{g})$ & $\mathrm{NR}^{\mathrm{c}}$ & $0.06 \pm 0.17$ & $0.08 \pm 0.15$ & $0.12 \pm 0.19$ \\
\hline
\end{tabular}

${ }^{\text {a }}$ Concentration units of $\mu \mathrm{g} \mathrm{m}^{-3}$ for MDL and precision are based on the IMPROVE volume of $32.8 \mathrm{~m}^{3}$. $^{\mathrm{b}}$ Value reported for network $(0.44 \mu \mathrm{g})$ in concentration units. ${ }^{\mathrm{c}}$ Not reported.
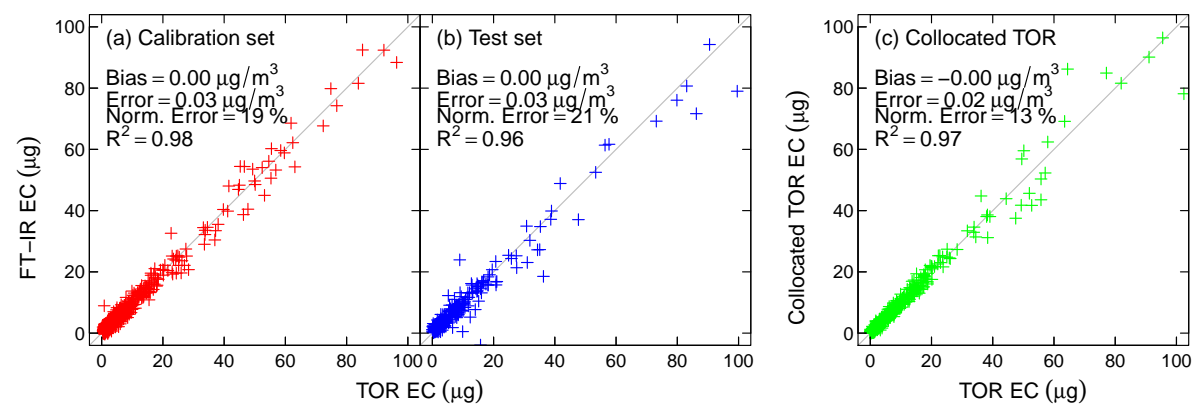

Figure 3. Predicted EC for calibration set (a) and test set (b) for the Base case using a hybrid calibration model. The collocated TOR samples (c) are from sites with parallel quartz filters that are both analyzed by TOR. Only the Phoenix site has samples in the calibration, test and collocated data sets. There are 521 samples in the calibration set (a), 265 samples in the test set (b) and 431 samples in the collocated TOR data set (c). Concentration units of $\mu \mathrm{g} \mathrm{m}^{-3}$ for bias and error are based on the IMPROVE nominal volume of $32.8 \mathrm{~m}^{3}$.

collocated TOR EC precision. The distribution of normalized errors for the calibration and test set for the raw spectra case and the collocated precision for the TOR samples are quite similar (Fig. S4a). The Base case bias $\left(0.00 \mu \mathrm{g} \mathrm{m}^{-3}\right)$ and absolute error $\left(0.03 \mu \mathrm{g} \mathrm{m}^{-3}\right)$ are on the same order as the Base case for TOR OC (test set bias $=0.02 \mu \mathrm{g} \mathrm{m}^{-3}$ and error $=0.08 \mu \mathrm{g} \mathrm{m}^{-3}$; Dillner and Takahama, 2015) and the $R^{2}$ values are the same (0.96; Dillner and Takahama, 2015). The normalized error for the test set $(21 \%)$ is higher than the collocated TOR EC precision $(13 \%)$ and higher than the TOR OC normalized error $(11 \%)$. The hybrid calibration also produces high-quality predictions of EC baselinecorrected and truncated spectra as indicated by the similarity in performance metrics for all three spectral types (Fig. S4b). The distribution of normalized errors for the calibration and test set for the baseline-corrected and truncated spectral pretreatments are similar to raw spectra and the collocated precision for the TOR samples (Fig. S4a). Section S5 demonstrates that the number of samples in the calibration set can be reduced and still provide high-quality predictions. The analysis suggests that the accuracy of FT-IR EC predictions is comparable to the precision of collocated TOR EC measurements.
Table 1 gives the MDL and collocated Phoenix sample precision for the hybrid FT-IR EC predictions for each spectral type and TOR EC. The MDL for all hybrid FT-IR EC spectral types are approximately the same as TOR EC with $3 \%$ or fewer of the samples below MDL. Section S5 shows that the MDL is independent of the number of blanks included in the calibration and the number of samples included in the calibration set (from two-thirds to one-third of the samples). The collocated Phoenix precision is better for the FT-IR methods than for TOR by about half. The mean predicted value for the blanks filters (last row of Table 1) is less than half of the first percentile of predicted EC values for the raw and baseline-corrected spectra and equivalent to the second percentile for the truncated case. The precision is on the same order for all three spectral types.

An alternate method for estimating EC is to predict total carbon $(\mathrm{TC}=\mathrm{OC}+\mathrm{EC})$ and subtract the $\mathrm{OC}$ prediction. This method can lead to higher errors on average than direct prediction of EC because the errors of both TC and OC are included in this method of estimation and these errors are larger than EC errors because EC mass is usually much less than OC mass. More discussion of this topic is included in Sect. S6. 


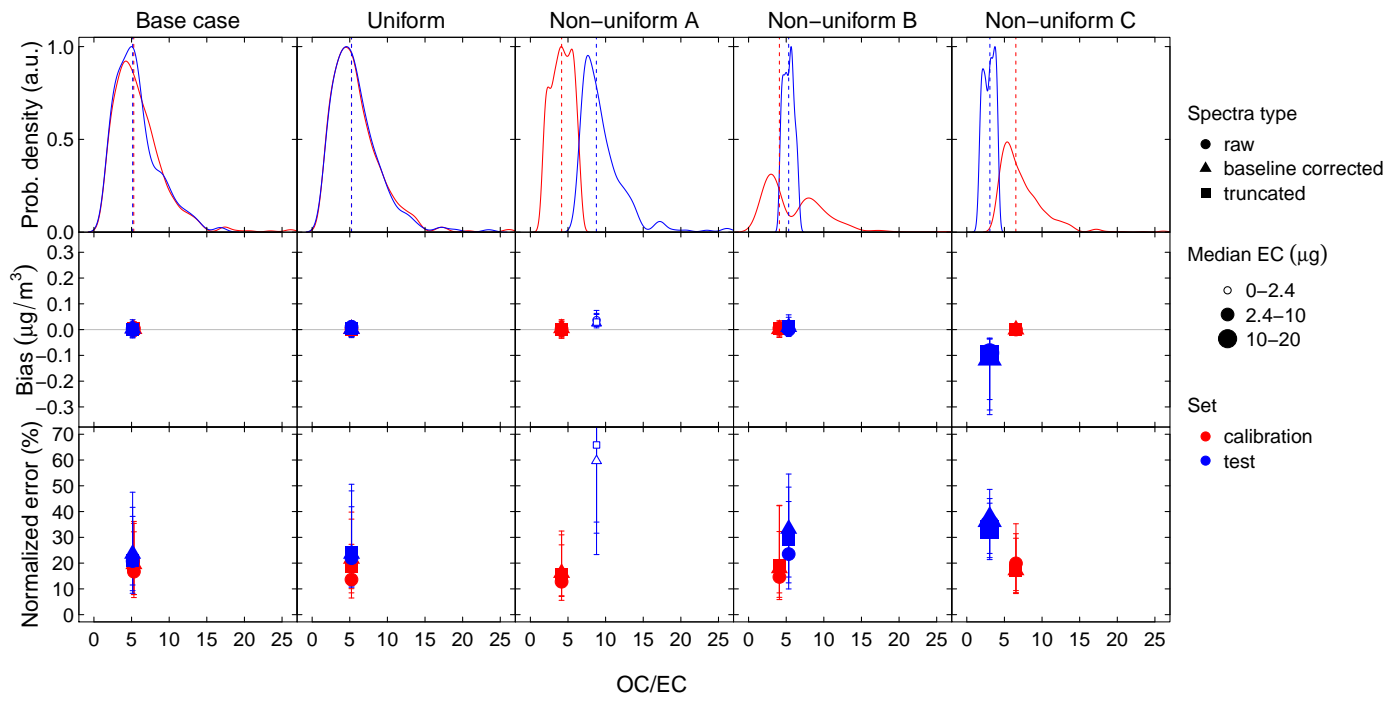

Figure 4. The probability distribution of $\mathrm{OC} / \mathrm{EC}$ and bias and normalized error (with the interquartile range shown by error bars) in the calibration (red) and test (blue) sets for five hybrid calibration cases: the Base case, the Uniform OC/EC case and three Nonuniform OC/EC cases. Vertical lines on the probability distributions are the color-coded median of the OC/EC distributions.

\subsection{Evaluating causes of bias and error by selecting the calibration and test sets based on measured parameters}

In this section, we consider the role of aerosol composition on the quantification of EC. Aerosol composition is described by the distribution of OC/EC and ammonium/EC. In other work, we show that $\mathrm{OC}$ and $\mathrm{EC}$ measurements by FT-IR and PLSR rely on similar wavebands for predictions (Takahama et al., 2015). Therefore, OC could be considered an interferant to the measurement of EC. We evaluate this possible interferant using the ratio of $\mathrm{OC}$ to $\mathrm{EC}$ because the impact of OC is likely dependent on its mass relative to EC mass. Ammonium absorbs FT-IR radiation in the same wave number region as $\mathrm{OC}$ and $\mathrm{EC}$ and so can also be considered an interferant. We use the ratio of ammonium to EC mass loadings to isolate the effect of ammonium. OC/EC and ammonium/EC are not correlated to 1/EC $\left(R^{2}<0.2\right)$, which indicates that impacts observed from EC, OC/EC and ammonium/EC are at least somewhat independent of each other. Therefore, separate calibrations were developed by ordering the samples by $\mathrm{OC} / \mathrm{EC}$ and ammonium/EC.

As was done for Uniform EC case, samples are arranged in ascending order by the parameter of interest prior to selection of filters for the calibration and test sets. Every third sample in the ordered list is put into the test set and the remaining samples are put into the calibration set. These cases are called the Uniform OC/EC case and Uniform ammonium/EC case. Three Nonuniform cases are also considered and the OC/EC Nonuniform cases are detailed here as an example: samples in the lowest two-thirds of the OC/EC range are used to predict samples in the highest third of the OC/EC range
(Nonuniform A); the highest and lowest third of the OC/EC range are used to predict the middle third OC/EC range (Nonuniform B); and the highest two-thirds of the OC/EC range are used to predict samples in the lowest third of the OC/EC range (Nonuniform C). All predictions are based on the hybrid calibration model (Sect. 3.1) such that Low EC samples in any of the test sets are predicted using a Low EC calibration developed for that case.

The top row of subplots in Fig. 4 shows that the distribution of $\mathrm{OC} / \mathrm{EC}$ in the test and calibration sets for the Base case and the Uniform OC/EC case are similar. The three Nonuniform OC/EC cases have distributions that are different for the test and calibrations sets. The Base and Uniform case have 0 bias and similar normalized error in the test and calibration sets indicating good predictions for these cases. When there is a large difference in OC/EC distribution between the test and calibration sets (Nonuniform cases), the normalized error is higher and sometimes a bias is induced. For Nonuniform $\mathrm{C}$, there is a significant negative bias and the normalized error is about $35 \%$ (10\% higher than the calibration set). For Nonuniform $\mathrm{B}$, in which the medians are similar but the distribution of $\mathrm{OC} / \mathrm{EC}$ is quite different between the sets, there is less than $10 \%$ higher error in the test set than the calibration set. For Nonuniform A, in which the median EC value is in the Low EC range, the median normalized error is at least $60 \%$ and the range of error is high, which is due to both the difference in the chemical composition of the aerosol in the test and calibration sets and the Low EC values.

The impact of ammonium is evaluated using Uniform and Nonuniform calibrations of ammonium/EC (Fig. 5). Like EC, the Base case, Uniform case and Nonuniform B case 


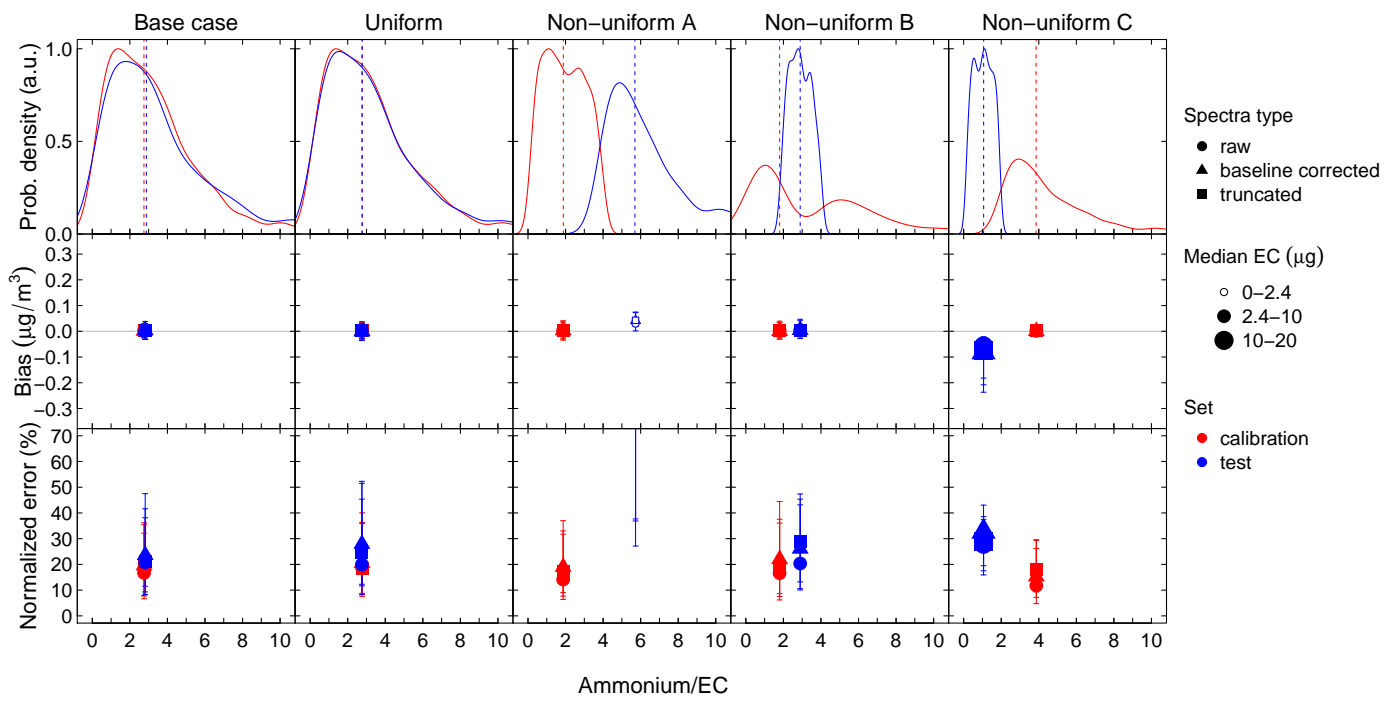

Figure 5. The probability distribution of ammonium/EC and bias and normalized error (with the interquartile range shown by error bars) in the calibration (red) and test (blue) sets for five hybrid calibration cases: the Base case, the Uniform ammonium/EC case and three Nonuniform ammonium/EC cases. Vertical lines on the probability distributions are the color-coded median of the ammonium/EC distributions.

have near 0 bias and low normalized error. The results for the Nonuniform A and C cases are very similar to the Nonuniform A case for OC/EC. In the Nonuniform $\mathrm{C}$ case, the calibration set contains a higher amount of ammonium and $\mathrm{EC}$ is under-predicted (some of the EC is assumed to be ammonium) in low ammonium/EC test samples (bias, -0.04 to $-0.06 \mu \mathrm{g} \mathrm{m}^{-3}$ ) and the range in bias of individual samples increases. The normalized error increases by about $15 \%$ from the calibration set to the test set but the error bars are about the same for the two sets. When low ammonium/EC samples are used to predict samples with high ammonium/EC (Nonuniform A), the normalized error becomes very large. This is due in part to the median EC value being below $2.4 \mu \mathrm{g}$. It is likely that additional error is induced because the calibration set is not trained to disregard ammonium in the prediction of EC, so some of the ammonium is reported to be EC leading to a positive bias and increased error. The distribution of EC, OC/EC and ammonium/EC for the test and calibration sets for the Base, Uniform and Nonuniform cases are shown in Sect. S7. When the chemical composition, as indicated by $\mathrm{OC} / \mathrm{EC}$ and ammonium/EC, is different between the calibration and test sets, the predictions have higher error than when the chemical compositions are similar.

\subsection{Prediction of specific sites}

Calibrations are developed using ambient samples for all but one site in the calibration set. The one site omitted from the calibration is predicted. Figure 6 shows the distributions of $\mathrm{EC}$ in the test and calibration set and the median and interquartile range of bias and normalized error for all sites. Three sites, Mesa Verde, Olympic and Trapper Creek, have median EC mass below $2.4 \mu \mathrm{g}$ and, although the bias is near
0 , they have the highest median (40 to $60 \%$ ) and range of normalized error. As shown with the Low EC calibration and comparison to collocated TOR samples (Sect. 3.1), these errors are similar to collocated precision of TOR samples in the same EC range, indicating that the error is due primarily to TOR analytical error. All other sites have higher EC mass and are expected to be predicted well, based on EC mass alone. However, only the Proctor Maple Research Facility is predicted well (Fig. 6). EC, OC/EC and ammonium/EC distributions at the Proctor Maple Research Facility are similar to the calibration set. The increased errors for the remaining three sites, Phoenix, Sac and Fox and St. Marks, are due to differences in aerosol composition between the calibration set and these sites. Distributions of EC, OC/EC and ammonium/EC for the test and calibration sets for all sites are shown in Sect. S7.

Figure 7 shows the OC/EC and ammonium/EC distributions at Phoenix and the ammonium/EC distributions for Sac and Fox and St. Marks. Phoenix, an urban site, has higher EC than the rural sites (Fig. 6). Phoenix also has lower OC/EC than the other sites (OC/EC Nonuniform $C)$ and has lower ammonium/EC than the rural sites (ammonium/EC Nonuniform C). All of these compositional differences lead to a negative bias and increased error as seen in the Phoenix samples (bias up to $0.03 \mu \mathrm{g} \mathrm{m}^{-3}$ and error up to $50 \%$ ). All six rural sites have similar OC/EC in the test set and the calibration set, indicating that $\mathrm{OC} / \mathrm{EC}$ does not impact the errors for these sites. Sac and Fox and St. Marks have higher ammonium/EC than the other sites (similar to ammonium/EC Nonuniform A except that the EC median is higher). As shown in Fig. 7, there is an increase range of bias values and higher error (22 to $40 \%$ ) in predictions for these sites 


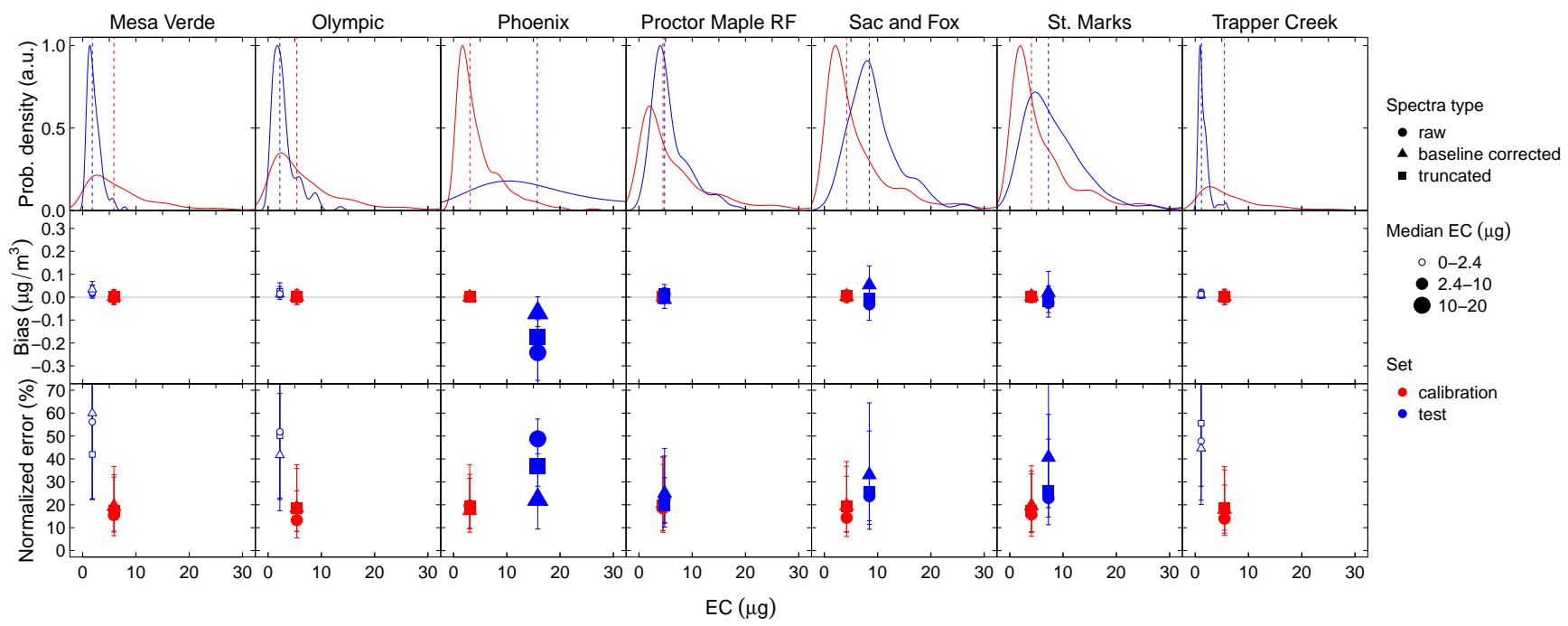

Figure 6. The distribution of EC and bias and normalized error (with the interquartile range shown by error bars) in the calibration (red) and test (blue) sets for calibrations developed for each site. Vertical lines are the median of the EC mass distributions color-coded for calibration and test sets.

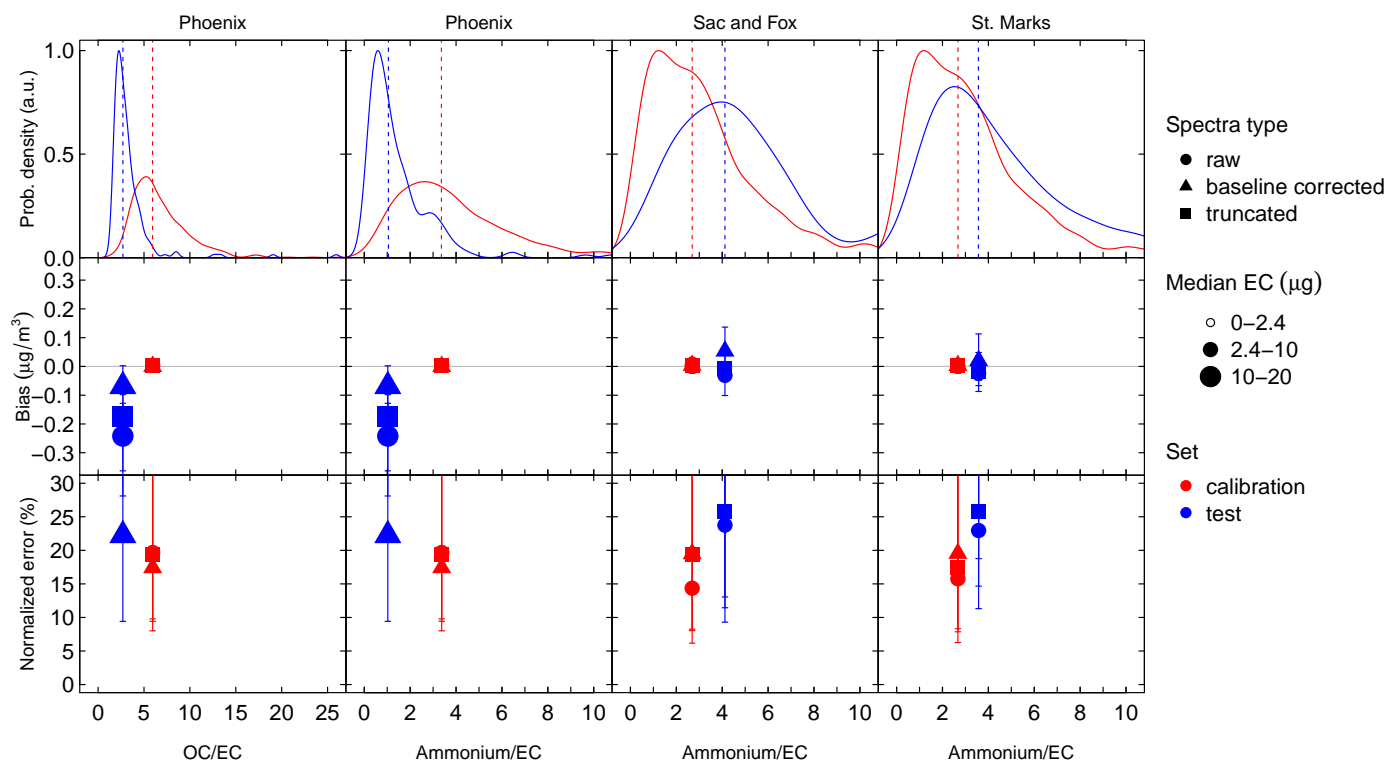

Figure 7. The distribution of OC/EC and bias and normalized error (with the interquartile range shown by error bars) in the calibration (red) and test (blue) sets for calibrations developed for Phoenix and the distribution of ammonium/EC, bias and normalized error for Phoenix, Sac and Fox and St. Marks. Vertical lines are the median of the distributions color-coded for calibration and test sets.

compared to the calibration set likely due to ammonium/EC differences.

We can therefore estimate how well a site not included in the calibration will be predicted based on the EC, OC/EC and ammonium/EC for the site. For sites with low EC mass, the error ranges from 40 to $60 \%$. Phoenix, which has higher $\mathrm{EC}$, lower OC/EC and lower ammonium/EC than the rural sites, has biased predictions and error between 20 and $50 \%$. Differences in ammonium/EC alone produce higher errors up to $40 \%$ for Sac and Fox and St. Marks, especially for the truncated spectral type.

\section{Conclusions}

Nondestructive, fast and inexpensive FT-IR analysis of routinely collected PTFE filters in the IMPROVE network can be used to predict TOR EC mass in IMPROVE aerosol samples. The FT-IR spectra and parallel TOR EC measurements 
are used in partial least squares regression to develop calibrations to predict TOR EC. A hybrid approach is used for calibration in which samples with Low EC are calibrated with a Low EC calibration and all other samples are calibrated with a calibration that spans the range of EC samples (a description of steps for calibration are included in the Supplement). The Low EC calibration produces predictions with mean error similar to that of collocated TOR samples in the same mass range and similar MDLs, indicating that the errors in the FT-IR method are primarily due to TOR measurement uncertainty. All three spectral types produce high-quality predictions. The hybrid calibrations developed from samples ordered by site date (Base case), EC, OC/EC and ammonium/EC, produce nearly bias-free predictions with low error $\left(\sim 0.02 \mu \mathrm{g} \mathrm{m}^{-3}\right.$ or $\left.20-25 \%\right)$. Blank filters in the test set are used to calculate MDL, but the number of blanks in the calibration set does not impact the value of the MDL. Using a calibration set that does not have similar composition to the test set (i.e., using samples in the calibration set that do not span the full range of EC, OC/EC or ammonium/OC) leads to higher bias and errors and is not recommended for obtaining high-quality predictions. In the proposed method, error and bias are kept to a minimum by including samples in the calibration set that have a similar composition as the test set, as indicated by EC, OC/EC and ammonium/EC. Therefore, we conclude that FT-IR spectra calibrated to TOR EC using partial least squares regression is a robust method for predicting TOR elemental carbon from particulate matter samples. Future work includes establishing that the calibration developed here can be used to predict TOR EC for samples collected during other years and developing a calibration that includes samples with a broader range of aerosol composition.

\section{The Supplement related to this article is available online at doi:10.5194/amt-8-4013-2015-supplement.}

Acknowledgements. The authors acknowledge funding from the IMPROVE program and EPA (National Park Service cooperative agreement P11AC91045) and EPFL startup funding.

Edited by: P. Herckes

\section{References}

Andreae, M. O. and Gelencsér, A.: Black carbon or brown carbon? The nature of light-absorbing carbonaceous aerosols, Atmos. Chem. Phys., 6, 3131-3148, doi:10.5194/acp-6-3131-2006, 2006.

Birch, M. E. and Cary, R. A.: Elemental carbon-based method for occupational monitoring of particulate diesel exhaust: Methodology and exposure issues, Analyst, 121, 1183-1190, doi:10.1039/an9962101183, 1996.
Bishop, C.: Pattern recognition and machine learning, SpringerVerlag, New York, 2006.

Bond, T. C. and Bergstrom, R. W.: Light absorption by carbonaceous particles: An investigative review, Aerosol Sci. Tech., 40, 27-67, doi:10.1080/02786820500421521, 2006.

Bond, T. C., Anderson, T. L., and Campbell, D.: Calibration and intercomparison of filter-based measurements of visible light absorption by aerosols, Aerosol Sci. Tech., 30, 582-600, doi:10.1080/027868299304435, 1999.

Bond, T. C., Doherty, S. J., Fahey, D. W., Forster, P. M., Berntsen, T., DeAngelo, B. J., Flanner, M. G., Ghan, S., Karcher, B., Koch, D., Kinne, S., Kondo, Y., Quinn, P. K., Sarofim, M. C., Schultz, M. G., Schulz, M., Venkataraman, C., Zhang, H., Zhang, S., Bellouin, N., Guttikunda, S. K., Hopke, P. K., Jacobson, M. Z., Kaiser, J. W., Klimont, Z., Lohmann, U., Schwarz, J. P., Shindell, D., Storelvmo, T., Warren, S. G., and Zender, C. S.: Bounding the role of black carbon in the climate system: A scientific assessment, J. Geophys. Res.-Atmos., 118, 5380-5552, doi:10.1002/jgrd.50171, 2013.

Bornemann, L. C., Kookana, R. S., and Welp, G.: Differential sorption behaviour of aromatic hydrocarbons on charcoals prepared at different temperatures from grass and wood, Chemosphere, 67, 1033-1042, doi:10.1016/j.chemosphere.2006.10.052, 2007.

Cavalli, F., Viana, M., Yttri, K. E., Genberg, J., and Putaud, J.-P.: Toward a standardised thermal-optical protocol for measuring atmospheric organic and elemental carbon: the EUSAAR protocol, Atmos. Meas. Tech., 3, 79-89, doi:10.5194/amt-3-79-2010, 2010.

Chow, J. C., Watson, J. G., Chen, L. W. A., Chang, M. C. O., Robinson, N. F., Trimble, D., and Kohl, S.: The IMPROVE-A temperature protocol for thermal/optical carbon analysis: maintaining consistency with a long-term database, J. Air Waste Manage. Assoc., 57, 1014-1023, doi:10.3155/1047-3289.57.9.1014, 2007.

Collaud Coen, M., Weingartner, E., Apituley, A., Ceburnis, D., Fierz-Schmidhauser, R., Flentje, H., Henzing, J. S., Jennings, S. G., Moerman, M., Petzold, A., Schmid, O., and Baltensperger, U.: Minimizing light absorption measurement artifacts of the Aethalometer: evaluation of five correction algorithms, Atmos. Meas. Tech., 3, 457-474, doi:10.5194/amt-3-457-2010, 2010.

Desert Research Intitute: DRI Model 2001 Thermal/Optical Carbon Analysis (TOR/TOT) of Aerosol Filter Samples - Method IMPROVE_A, Desert Research Institute, Standard Operating Procedure, Reno, Nevada, 2012.

Dillner, A. M. and Takahama, S.: Predicting ambient aerosol thermal-optical reflectance (TOR) measurements from infrared spectra: organic carbon, Atmos. Meas. Tech., 8, 1097-1109, doi:10.5194/amt-8-1097-2015, 2015.

Flanagan, J. B., Jayanty, R. K. M., Rickman, J. E. E., and Peterson, M. R.: $\mathrm{PM}_{2.5}$ Speciation Trends Network: Evaluation of Whole-System Uncertainties Using Data from Sites with Collocated Samplers, J. Air Waste Manage. Assoc., 56, 492-499, doi:10.1080/10473289.2006.10464516, 2006.

Friedel, R. A. and Carlson, G. L.: Infrared Spectra of Ground Graphite, J. Phys. Chem., 75, 1149, doi:10.1021/j100678a021, 1971.

Hand, J. L., Schichtel, B. A., Pitchford, M., Malm, W. C., and Frank, N. H.: Seasonal composition of remote and urban fine particulate matter in the United States, J. Geophys. Res.-Atmos., 117, D05209, doi:10.1029/2011jd017122, 2012. 
Hand, J. L., Schichtel, B. A., Malm, W. C., and Frank, N. H.: Spatial and Temporal Trends in $\mathrm{PM}_{2.5}$ Organic and Elemental Carbon across the United States, Adv. Meteorol., 2013, 367674, doi:10.1155/2013/367674, 2013.

Hand, J. L., Schichtel, B. A., Malm, W. C., Copeland, S., Molenar, J. V., Frank, N., and Pitchford, M.: Widespread reductions in haze across the United States from the early 1990s through 2011, Atmos. Environ., 94, 671-679, doi:10.1016/j.atmosenv.2014.05.062, 2014.

Hastie, T., Tibshirani, R., and Friedman, J.: The Elements of Statistical Learning - Data Mining, Inference, and Prediction, Second Edition, 2nd Edn., Springer Series in Statistics, Springer, New York, 745 pp., 2009.

Hidy, G. M., Blanchard, C. L., Baumann, K., Edgerton, E., Tanenbaum, S., Shaw, S., Knipping, E., Tombach, I., Jansen, J., and Walters, J.: Chemical climatology of the southeastern United States, 1999-2013, Atmos. Chem. Phys., 14, 11893-11914, doi:10.5194/acp-14-11893-2014, 2014.

Janssen, N. A. H., Hoek, G., Simic-Lawson, M., Fischer, P., van Bree, L., ten Brink, H., Keuken, M., Atkinson, R. W., Anderson, H. R., Brunekreef, B., and Cassee, F. R.: Black Carbon as an Additional Indicator of the Adverse Health Effects of Airborne Particles Compared with $\mathrm{PM}_{10}$ and $\mathrm{PM}_{2.5}$, Environ. Health Perspect., 119, 1691-1699, doi:10.1289/ehp.1003369, 2011.

Krall, J. R., Anderson, G. B., Dominici, F., Bell, M. L., and Peng, R. D.: Short-term Exposure to Particulate Matter Constituents and Mortality in a National Study of US Urban Communities, Environ. Health Perspect., 121, 1148-1153, doi:10.1289/ehp.1206185, 2013.

Liu, X., Easter, R. C., Ghan, S. J., Zaveri, R., Rasch, P., Shi, X., Lamarque, J. F., Gettelman, A., Morrison, H., Vitt, F., Conley, A., Park, S., Neale, R., Hannay, C., Ekman, A. M. L., Hess, P., Mahowald, N., Collins, W., Iacono, M. J., Bretherton, C. S., Flanner, M. G., and Mitchell, D.: Toward a minimal representation of aerosols in climate models: description and evaluation in the Community Atmosphere Model CAM5, Geosci. Model Dev., 5, 709-739, doi:10.5194/gmd-5-709-2012, 2012.

Malm, W. C., Sisler, J. F., Huffman, D., Eldred, R. A., and Cahill, T. A.: Spatial and seasonal trends in particle concentration and optical extinction in the United-States, J. Geophys. Res.-Atmos., 99, 1347-1370, doi:10.1029/93jd02916, 1994.

Mao, Y. H., Li, Q. B., Zhang, L., Chen, Y., Randerson, J. T., Chen, D., and Liou, K. N.: Biomass burning contribution to black carbon in the Western United States Mountain Ranges, Atmos. Chem. Phys., 11, 11253-11266, doi:10.5194/acp-1111253-2011, 2011.

Mevik, B.-H. and Cederkvist, H. R.: Mean squared error of prediction (MSEP) estimates for principal component regression (PCR) and partial least squares regression (PLSR), J. Chemometr., 18, 422-429, doi:10.1002/cem.887, 2004.
Mevik, B. H. and Wehrens, R.: The pls package: Principal component and partial least squares regression in R, J. Stat. Softw., 18, 1-24, 2007.

Petzold, A., Schloesser, H., Sheridan, P. J., Arnott, W. P., Ogren, J. A., and Virkkula, A.: Evaluation of multiangle absorption photometry for measuring aerosol light absorption, Aerosol Sci. Tech., 39, 40-51, doi:10.1080/027868290901945, 2005.

Polshin, E., Aernouts, B., Saeys, W., Delvaux, F., Delvaux, F. R., Saison, D., Hertog, M., Nicolai, B. M., and Lammertyn, J.: Beer quality screening by FT-IR spectrometry: Impact of measurement strategies, data pre-processings and variable selection algorithms, J. Food Eng., 106, 188-198, doi:10.1016/j.jfoodeng.2011.05.003, 2011.

R Core Team: R: A Language and Environment for Statistical Computing, R Foundation for Statistical Computing, Vienna, Austria, 2014.

Ruthenburg, T. C., Perlin, P. C., Liu, V., McDade, C. E., and Dillner, A. M.: Determination of organic matter and organic matter to organic carbon ratios by infrared spectroscopy with application to selected sites in the IMPROVE network, Atmos. Environ., 86 , 47-57, doi:10.1016/j.atmosenv.2013.12.034, 2014.

Smith, D. M., Griffin, J. J., and Goldberg, E. D.: Spectroscopic method for quantitative-determination of elemental carbon, Abstracts of Papers of the American Chemical Society, 116-117, 1975.

Takahama, S., Johnson, A., and Russell, L. M.: Quantification of Carboxylic and Carbonyl Functional Groups in Organic Aerosol Infrared Absorbance Spectra, Aerosol Sci. Tech., 47, 310-325, doi:10.1080/02786826.2012.752065, 2013.

Takahama, S., Ruggeri, G., and Dillner, A. M.: Quantification of organic functional groups in atmospheric aerosols by infrared spectroscopy: interpretation of multivariate calibration models through sparse methods, in preparation, 2015.

Torseth, K., Aas, W., Breivik, K., Fjaeraa, A. M., Fiebig, M., Hjellbrekke, A. G., Myhre, C. L., Solberg, S., and Yttri, K. E.: Introduction to the European Monitoring and Evaluation Programme (EMEP) and observed atmospheric composition change during 1972-2009, Atmos. Chem. Phys., 12, 5447-5481, doi:10.5194/acp-12-5447-2012, 2012.

Weakley, A. T., Miller, A. L., Griffiths, P. R., and Bayman, S. J.: Quantifying silica in filter-deposited mine dusts using infrared spectra and partial least squares regression, Anal. Bioanal. Chem., 406, 4715-4724, doi:10.1007/s00216-014-7856-y, 2014.

White, W. H., Trzepla, K., and Hyslop, N. P.: Light Absorption by Aerosol Deposits on PTFE Filters: A Decade of BackscatterCorrected Measurements by the IMPROVE Network, in preparation, 2015.

Witten, I. H., Frank, E., and Hall, M. A.: Data Mining: Practical Machine Learning Tools and Techniques, 3rd Edn., Morgan Kaufmann Publishers Inc., San Francisco, CA, USA, 2011. 